\title{
Episodic memory function is associated with multiple measures of white matter integrity in cognitive aging
}

\section{Samuel N. Lockhart, 1,2,3* Adriane B. V. Mayda ${ }^{1,2,3}$, Alexandra E. Roach ${ }^{1,2,4}$, Evan Fletcher ${ }^{1,2}$, Owen Carmichael ${ }^{1,2}$, Pauline Maillard ${ }^{1,2}$, Christopher G. Schwarz ${ }^{1,2}$, Andrew P. Yonelinas ${ }^{3,4}$, Charan Ranganath ${ }^{3,4}$ and Charles DeCarli ${ }^{1,2,3,4}$}

\author{
Imaging of Dementia and Aging Lab, Center for Neuroscience, University of California Davis, Davis, CA, USA \\ 2 Department of Neurology, University of California Davis, Davis, CA, USA \\ ${ }^{3}$ Neuroscience Graduate Group, University of California Davis, Davis, CA, USA \\ ${ }^{4}$ Department of Psychology, University of California Davis, Davis, CA, USA
}

\section{Edited by:}

Simone Rossi, Azienda Ospedalira

Universitaria Senese, Italy

Reviewed by:

Cheryl Grady, University of Toronto,

Canada

Alexa Morcom, University of

Edinburgh, UK

*Correspondence:

Samuel N. Lockhart, Imaging of Dementia and Aging Lab, Center for

Neuroscience, University of

California Davis, 1544 Newton

Court, Davis, CA 95618, USA.

e-mail: snlockhart@ucdavis.edu
Previous neuroimaging research indicates that white matter injury and integrity, measured respectively by white matter hyperintensities (WMH) and fractional anisotropy (FA) obtained from diffusion tensor imaging (DTI), differ with aging and cerebrovascular disease (CVD) and are associated with episodic memory deficits in cognitively normal older adults. However, knowledge about tract-specific relationships between WMH, FA, and episodic memory in aging remains limited. We hypothesized that white matter connections between frontal cortex and subcortical structures as well as connections between frontal and temporo-parietal cortex would be most affected. In the current study, we examined relationships between WMH, FA and episodic memory in 15 young adults, 13 elders with minimal $\mathrm{WMH}$ and 15 elders with extensive $\mathrm{WMH}$, using an episodic recognition memory test for object-color associations. Voxel-based statistics were used to identify voxel clusters where white matter measures were specifically associated with variations in episodic memory performance, and white matter tracts intersecting these clusters were analyzed to examine white matter-memory relationships. White matter injury and integrity measures were significantly associated with episodic memory in extensive regions of white matter, located predominantly in frontal, parietal, and subcortical regions. Template based tractography indicated that white matter injury, as measured by $\mathrm{WMH}$, in the uncinate and inferior longitudinal fasciculi were significantly negatively associated with episodic memory performance. Other tracts such as thalamo-frontal projections, superior longitudinal fasciculus, and dorsal cingulum bundle demonstrated strong negative associations as well. The results suggest that white matter injury to multiple pathways, including connections of frontal and temporal cortex and frontal-subcortical white matter tracts, plays a critical role in memory differences seen in older individuals.

Keywords: aging, cerebrovascular disease, magnetic resonance imaging, white matter hyperintensity, fractional anisotropy, structural connectivity, episodic memory, source memory

\section{INTRODUCTION}

Cognitive aging research reveals that even healthy, cognitively "normal" older adults show subtle performance differences with increasing age on many cognitive tasks, including tasks tapping speed, spatial visualization, executive control, and episodic memory encoding and retrieval (Grady and Craik, 2000; Nordahl et al., 2006; Salthouse, 2009; Dickerson and Eichenbaum, 2010). Understanding the causes of such age-related differences in cognitive function remains a challenge, and several theories attempt to explain mediators of cognitive differences in terms of localized changes in brain structure and function. One such theory, the frontal aging hypothesis, posits that executive control deficits associated with prefrontal cortex (PFC) dysfunction mediate normal age-related cognitive differences (West, 1996). According to this hypothesis, the human frontal lobes, particularly PFC subregions, are especially vulnerable to age-related degeneration, and functions dependent on frontal regions, such as executive control, are selectively impaired with age. Therefore, executive control deficits associated with frontal dysfunction are postulated to mediate normal age-related cognitive differences, including memory differences.

Current theories of episodic memory encoding and retrieval emphasize age-related differences in frontal systems and their role in successful performance with age (Cabeza, 2002; Buckner, 2004; Bollinger et al., 2011). Source memory, the ability to recollect the context in which an event took place or how one acquired episodic knowledge of an event, can be particularly vulnerable to the effects of aging (Glisky et al., 2001). Further, behavioral and electrophysiological studies of patients with PFC lesions demonstrate that frontal lobe damage can selectively impair source memory independent of item memory (Janowsky et al., 1989; Duarte et al., 2005; Swick et al., 2006), suggesting that frontal function is 
crucial for item-context binding and successful episodic memory performance. Recently, functional magnetic resonance imaging (fMRI) studies have also supported the idea that age differences in PFC activation (particularly lateral PFC) may be related to source memory deficits (Rajah et al., 2010; Dulas and Duarte, 2012). However, considering recent evidence regarding networkspecific effects of neurobiological changes on cognition (Ziegler et al., 2010) and advances in the delineation of connections of the PFC in human and monkey studies (Mori et al., 2005; Schmahmann and Pandya, 2006; Yeterian et al., 2012), the frontal aging hypothesis may be insufficient for conceptualizing the complex effects aging has on cognitive functions dependent on distributed brain networks. In order to overcome such limitations, newer approaches must consider the effects of aging on the integrity of several interrelated processing networks spanning multiple regions of the brain.

We hypothesize that age-related differences in episodic memory performance may result from alterations in white matter integrity - related to not only age but also coincident vascular disease-that may affect the connectivity of networks important to cognition. We are specifically interested in how changes to anatomically specific white matter tracts connecting distributed brain regions, particularly connections of the frontal lobe with its targets, may contribute to changes in episodic memory function.

Degenerative white matter changes have been shown to occur in both normal aging and neurological disease (Pfefferbaum et al., 2000; Vernooij et al., 2008; Lee et al., 2010). White matter abnormalities linked to cardiovascular risk factors and cerebrovascular disease (CVD), measured by white matter hyperintensities (WMH) seen on MRI, occur even during normal aging and increase in extent with age (DeCarli et al., 1995, 2005a,b). Both $\mathrm{WMH}$ and subtler changes to white matter integrity, measured by the diffusion tensor imaging (DTI) parameter of fractional anisotropy (FA), have been shown to mediate age-related differences in cognitive performance, possibly due to disconnection of distributed cognitive networks (Nordahl et al., 2006; AndrewsHanna et al., 2007; Jacobs et al., 2011). These measures are thought to index different aspects of white matter integrity, with WMH representing "core" regions of vascular white matter injury, while FA reductions in surrounding normal-appearing white matter indicate more subtle changes in white matter integrity associated with microstructural alterations (Assaf and Pasternak, 2008; Lee et al., 2010; Maillard et al., 2011).

Previous research indicates that WMH are associated with impairments in episodic memory, working memory, and executive control among cognitively normal older adults (Nordahl et al., 2005, 2006; Mayda et al., 2011). Further, executive control deficits seen in subjects with extensive WMH are hypothesized to contribute to episodic memory deficits (Parks et al., 2011). These results support the hypothesis that white matter tract injury, by disconnecting frontal areas from distributed systems necessary for task-relevant cognitive performance, may be a neural mechanism associated with age-related control impairments and PFC dysfunction.

However, previous studies relating white matter structural changes in prefrontal and other brain areas to age-related differences in episodic memory performance have not identified which specific white matter tracts are most involved in network disconnection. One previous study has directly tested the hypothesis that cognitive performance in older adults correlates more strongly with white matter than gray matter changes, demonstrating associations between episodic memory performance and FA in temporal and posterior parietal areas, and advancing the idea that disconnection may be important to cognitive differences (Ziegler et al., 2010). Similar to such recent studies from other labs, we predict that white matter integrity in anatomically specific regions will be associated with cognition. To test this hypothesis, we examined regional associations between white matter injury and integrity measures and episodic source memory performance in healthy, cognitively normal older and young adult subjects. Specifically, we hypothesize that particular white matter connections of the frontal lobe with its targets would be most affected (Nordahl et al., 2006; Schott et al., 2011; Smith et al., 2011; Yeterian et al., 2012), particularly reciprocal long cortico-cortical connections between prefrontal and parietal cortex (Goldman-Rakic, 1987; Selemon and Goldman-Rakic, 1988; Cavada and Goldman-Rakic, 1989), as well as connections between frontal cortex and subcortical structures (Alexander et al., 1986; Cummings, 1993), and between frontal and temporal cortex (Cabeza and Nyberg, 2000; Nordahl et al., 2006). We hypothesize that network disconnection due to white matter changes, as measured by WMH and DTI, will be associated with age-related differences in episodic memory performance.

\section{METHODS \\ PARTICIPANTS}

Three groups of adults were included in the current study. Healthy, cognitively normal older adults were recruited from a pool of normal control individuals seen at the UC Davis Alzheimer's Disease Center (ADC) and subdivided into high and low WMH volume groups, and young adult controls were recruited by advertisement from the university. High WMH volume was defined as the highest quartile of WMH volumes among all normal subjects seen at the ADC, with low WMH volume defined as the lowest quartile of normal ADC subjects. WMH group classification was based on imaging data from a previous scan. Eighteen high WMH, 15 low WMH, and 15 young adults participated in this research. Prior to analyses, data from three high WMH subjects were excluded, one due to the presence of a silent stroke, and two due to memory and neuropsychological task non-compliance. In addition, data from two low WMH subjects were excluded, one due to fluid-attenuated inversion recovery (FLAIR) image abnormalities, and another due to task non-compliance.

Thus, for the global analyses relating cognition to white matter integrity (using FLAIR and WMH), we analyzed data from 43 total subjects (Table 1): 15 young adult controls (aged 19-29), 13 low WMH subjects (aged 66-84) and 15 high WMH subjects (aged 66-89). The two older adult groups did not significantly differ in age. A further two subjects, one young adult and one high WMH subject, were excluded from analyses of FA-cognition relationships due to artifacts in the image and poor quality scans (FA analysis $n=41$ ). 
Table 1 | Subject demographic information.

\begin{tabular}{llll}
\hline & Young & Low WMH & High WMH \\
\hline $\mathrm{N}$ & 15 & 13 & 15 \\
Mean (SD) age & $24.1(3.1)^{a}$ & $75.5(4.7)$ & $77.3(5.9)$ \\
Mean (SD) years of education & $16.0(2.0)$ & $15.6(1.8)$ & $11 / 2$ \\
Sex (F/M) & $6 / 9$ & $29.3(0.75)$ & $9 / 6$ \\
Mean (SD) MMSE score & $\mathrm{N} / \mathrm{A}$ & 38 & $28.7(1.2)^{b}$ \\
\% Minority & 40 & $10.8(1.3)$ & 53 \\
Mean (SD) forward digit span & $11(1.9)$ & $7.2(2.3)$ & $9.8(1.9)$ \\
Mean (SD) backward digit span & $7.9(2.1)$ & $21.9(6.5)^{d}$ & $5.9(1.8)^{c}$ \\
Mean (SD) category fluency & $27.1(5.9)^{d}$ & $16.3(3.1)^{d}$ \\
\hline
\end{tabular}

a Differs from all groups, $p<0.0001$;

${ }^{b}$ High WMH marginally differ from Low WMH, $p=0.09$;

${ }^{c}$ Differs from young, $p<0.008$;

${ }^{d}$ All groups differ, $p<0.04$.

The cognitively normal older adults in this study were in stable health, recruited both from a pool of normal control individuals seen for evaluation at the ADC, and from the community through advertising or from spouses of patients. The diagnosis of cognitively normal was made following a clinical neurological exam and neuropsychological work up, which included Mini Mental State Exam (MMSE), Wechsler Memory Scale-Revised, Logical Memory I and II, Memory Assessment Scales List Learning, Boston Naming, Block Design, Digit Span, Animal Fluency, and Geriatric Depression Scale. This diagnosis was adjudicated at a multidisciplinary case conference, based upon all available clinical information. Exclusion criteria were limited to diagnosis of dementia or mild cognitive impairment, major medical illness, history of cortical strokes, medications thought to affect cognition, and WMH volume (based on a previous scan) outside the ranges defined in the group selection criteria above. The Institutional Review Board of the University of California Davis approved the study, and informed consent was obtained from all subjects.

\section{MRI METHODS}

\section{MRI data acquisition and preprocessing}

Structural magnetic resonance imaging was conducted on a General Electric 1.5T Signa Horizon LX NV/I System. Wholebrain imaging sequences included a T1-weighted, coronal 3D spoiled gradient recalled echo sequence (3DSPGR, IR-prepped, $\mathrm{TE}=1.9 \mathrm{~ms}$, flip angle $=20^{\circ}$, field of view $(\mathrm{FOV})=24 \mathrm{~cm}$, matrix $=256 \times 256,124$ contiguous slices, slice thickness $=$ $1.6 \mathrm{~mm}$ ), as well as an axial-oblique 2D FLAIR fast spin echo sequence $(\mathrm{TE}=144 \mathrm{~ms}, \mathrm{TR}=11,000 \mathrm{~ms}, \mathrm{TI}=2250 \mathrm{~ms}$, flip angle $=90^{\circ}, \mathrm{FOV}=22 \mathrm{~cm}$, matrix $=256 \times 192$, contiguous slices, slice thickness $=3 \mathrm{~mm}$ ). DTI data were collected using single-shot spin-echo echo-planar imaging $(\mathrm{TE}=94 \mathrm{~ms}, \mathrm{TR}=$ $8000 \mathrm{~ms}$, flip angle $=90^{\circ}, \mathrm{FOV}=22 \mathrm{~cm}$, matrix $=128 \times 128$, slice thickness $=6 \mathrm{~mm}, b$-value $=1000 \mathrm{~s} / \mathrm{mm}^{2}, 6$ gradient directions, $25 \mathrm{~ms}$ time between diffusion gradient pulses).

We preprocessed images and quantified total brain and total WMH volumes for all subjects using custom-written computer programs operating on a LINUX platform as previously described
(DeCarli et al., 2005b). Non-brain tissue was removed from all images, and image intensity non-uniformity correction applied (DeCarli et al., 1996). Once brain matter segmentation was achieved, a segmentation threshold for WMH volume on FLAIR was determined a priori as 3.5 standard deviations in pixel intensity above the mean of the fitted distribution of brain parenchyma. Repeated estimates using inter-class correlations have shown values around 0.98 for brain segmentation (DeCarli et al., 2005b). Morphometric erosion of two exterior image pixels was applied to the brain matter image before modeling to remove the effects of partial volume cerebrospinal fluid pixels on white matter hyperintensity determination (DeCarli et al., 1992, 1996). Highly trained analysts blinded to subject age, sex, and group status performed all MRI analyses. Inter-rater reliabilities for these are repeatedly calculated and are consistently above 0.90 for all measures.

Multi-modal mapping and analysis of WMH and DTI were performed as an extension of previously published methods. Briefly, all images were non-linearly aligned to a common stereotaxic space to allow precise colocalization of WMH and FA, particularly in the cerebral white matter (see appendix in DeCarli et al., 2005a). Here we will use the term white matter injury to indicate the presence of $\mathrm{WMH}$, while white matter integrity refers to changes measured by both WMH and DTI.

\section{FLAIR image intensity normalization}

For voxel-based regression analyses, we rescaled all FLAIR images to a common range of values (0-255) and aligned the peaks of all image intensity histograms. This had the effect of normalizing the image intensity distributions, so that FLAIR signal values were comparable from subject to subject.

\section{Diffusion tensor imaging}

Diffusion tensor imaging was conducted using a diffusion tensor-weighted echo-planar sequence (Stanford University, Stanford, CA). Six gradient directions, acquired twice at both reversed and non-reversed gradient polarity for a total of four images per direction, were used to generate diffusionweighted images. DTI data processing, including susceptibility 
artifact correction, was done using FSL command line utilities (http://www.fmrib.ox.ac.uk/fsl/).

After distortion correction, the four images from each gradient direction were averaged to produce six spatially registered diffusion weighted images. The two non-diffusion weighted images were also averaged to produce a seventh image for processing and generation of diffusion tensor images, according to the theory and methods detailed by Basser (Basser and Pierpaoli, 1998). Generating images from gradient combinations and from the same combinations with reversal of gradient polarity produced averaged images with fewer distortions. This is due to image distortion cancellation from higher order residual magnetic fields produced by eddy currents generated from the interactions of switching of multiple gradient fields. Finally, diffusion tensor images were computed from the seven averaged directional images, providing a diffusion tensor at each voxel, a matrix representation of the local directional statistics of water diffusion. FA was then calculated at each image voxel.

\section{Hopkins probabilistic tractography atlas analyses}

To further understand specific white matter tracts that may be injured by CVD as manifest by WMH, we used probabilistic maps of the location of multiple fiber tracts, obtained from automated tractography on multiple subjects (Zhang et al., 2010). Such probabilistic fiber map atlases are made publicly available for research use (http://cmrm.med.jhmi.edu). Each fiber tract template file provides a value at each voxel in MNI space representing the probability of the tract passing through that voxel. Tract probability maps were aligned and warped to the same common anatomical template space as subject FA and WMH maps. Tracts were then overlaid on subject WMH and FA images in order to calculate log-normalized WMH volumes and mean FA values for the entire length of each tract of interest.

\section{COGNITIVE TESTING}

Subjects were administered an episodic recognition memory task outside the scanner in a single session using E-Prime software on a laptop computer at the UC Davis Imaging Research Center. Previous studies have demonstrated impaired episodic source memory accuracy in aging and in patients with PFC lesions, and frontal function is considered important for associating an item with the context in which it was learned (Janowsky et al., 1989; Glisky et al., 2001; Duarte et al., 2005; Swick et al., 2006). The color-picture association task has been shown to be sensitive to prefrontal and hippocampal function, and is thought to tap episodic memory encoding and retrieval processes (Yonelinas et al., 2001). In the self-paced study phase prior to scanning, subjects viewed 40 centrally presented images of familiar objects, with half in red, and half in green (Figure 1), and were asked to form an association between the color and the object, thus providing a verbal encoding strategy. At least $2 \mathrm{~h}$ later (post scanning session), previously studied objects were presented in black every $4000 \mathrm{~ms}$ and subjects were asked to indicate by button press whether the images were previously presented in green or red. Total episodic accuracy (percent correct) was measured and entered into analyses. Measures of fluency and working memory were also used. The working memory measures included

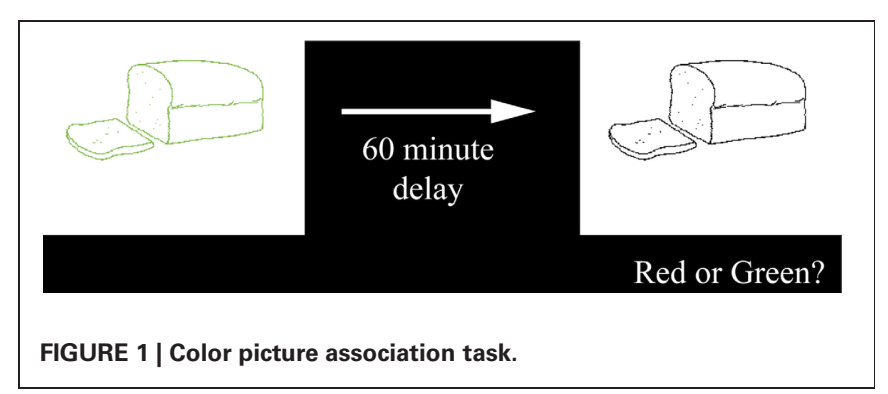

digit span forward and backward, and the fluency measure was Category Fluency (number of animals named in $60 \mathrm{~s}$ ).

\section{STATISTICAL ANALYSES}

Statistical analyses of imaging and cognitive data were conducted at multiple levels: at the global white matter level, at the voxel level, and for specific tract regions of interest (ROIs). Voxel-wise regressions were conducted using $\mathrm{C}++$ applications written for the LINUX platform; additional statistical analyses were conducted using the $\mathrm{R}$ statistical computing language (R Development Core Team, 2011).

First, global measures of white matter integrity data, including global WMH volume $(n=43)$ normalized to head size and logtransformed (DeCarli et al., 2008), and mean global white matter FA $(n=41)$, were regressed against memory performance. Since FLAIR and WMH measures are highly correlated, we focused our global analyses on WMH; using FLAIR measures did not materially change the global results. All subject images (3DT1, FLAIR, $\mathrm{WMH}$, and FA images) were then aligned and warped into a common stereotaxic space, to allow for anatomical localization of differences in white matter integrity related to cognition. One of the authors (E.F.) developed C++ applications for voxel-based multiple regression analyses. These measure the amount of variance explained in a cognitive variable by a linear combination of weighted terms of an imaging signal variable at each voxel (FLAIR or FA) and covariates of no interest (age, sex, education):

$$
Y=\beta_{1} \times J_{x, y, z}+\beta_{2} \times \text { age }+\beta_{3} \times \text { sex }+\beta_{4} \times \text { education }+\varepsilon
$$

where $Y=$ episodic memory performance and $J$ is the imaging modality measured at voxel location $x, y, z$.

Regressions were limited to voxels within a cerebral white matter mask, which encompassed white matter voxels common to all subjects. For these analyses, we used normalized FLAIR intensity levels as a continuous measure of white matter injury (as $\mathrm{WMH}$ values are binary at each voxel), and FA values as a continuous measure of white matter integrity. Values representing the strength of the relationship at each voxel ( $t$-scores), over the entire brain, which passed a predetermined threshold of $t \pm 2$ (sign dependent on expected direction of relationship) were then entered into non-parametric significance testing (5000 permutations) for significant-sized clusters of correlations (Nichols and Holmes, 2002). All clusters which then passed a criterion of $p<$ 0.05 in this cluster level permutation analysis were selected and displayed on images along with brain slices and ventricles for spatial reference. 
Finally, in order to identify which tracts would demonstrate anatomically specific relationships between white matter integrity and memory performance, we extracted white matter integrity measures from selected tracts, with the aid of the probabilistic fiber map atlas described above. Of particular interest were fibers intersecting the clusters identified in the cluster analyses. These tract-based white matter integrity measures (bilateral total log-normalized WMH volume and bilateral mean FA) were then entered into regression analyses as predictors of cognitive performance, including covariates of age, sex, and education. We analyzed white matter integrity measures along the entirety of each tract. Again, as FLAIR and WMH measures are highly correlated, we focused our tract analyses on WMH as WMH are the most theoretically relevant measure; using FLAIR measures did not materially change the results. In addition, other tracts outside the cluster were selected and tested to confirm the specificity of associations within the cluster. We performed tests to demonstrate not only that certain tracts and not others were related to episodic memory performance, but also that other behaviors were not associated with white matter integrity in the same tracts. Analyses were performed for subjects that had both $\mathrm{WMH}$ and FA images. To control the familywise error rate at $p<0.05$ for each of 10 tract-based models, white matter integrity measures were only considered significant if they passed a per-comparison threshold of $p<0.00512$.

\section{RESULTS \\ GLOBAL WHITE MATTER INJURY AND INTEGRITY DIFFER BY SUBJECT GROUP}

As expected based on the selection parameters of the study, ANOVA and $t$-tests confirmed differences in log-normalized WMH volume among the subject groups $[F(2,40)=211.7, p<$ 0.0001; Figure 2A]. High WMH older adults (whose group classification was based on a previous scan) indeed had higher WMH volume on this scan than both low WMH older adults $(p<$ $0.0001)$ and young adults $(p<0.0001)$, and low WMH older adults had higher WMH volume than young adults $(p<0.0001)$. We also confirmed mean FA differences among the subject groups $[F(2,38)=48.3, p<0.0001$; Figure $2 B]$ with younger adults having higher mean FA than high WMH $(p<0.0001)$ and low
WMH $(p<0.0001)$ older adults, and low WMH older adults having higher mean FA than high WMH older adults $(p=0.002)$. Additionally, across all subjects, global WMH volume and mean FA were significantly negatively correlated (Figure 2C; $R^{2}=0.63$, $p<0.0001)$. Mean normalized white matter FLAIR and lognormalized WMH were also highly positively correlated $\left(R^{2}=\right.$ $0.85, p<0.0001)$, and as we found no differences when using FLAIR instead of WMH, we report only tests using WMH for our global and tract-specific analyses.

\section{EPISODIC MEMORY IS ASSOCIATED WITH GLOBAL WHITE MATTER INJURY AND INTEGRITY}

ANOVA and $t$-tests confirmed differences in episodic memory performance among the subject groups $[F(2,40)=22.7, p<$ 0.0001 ; Figure 3A] with younger adults having higher episodic accuracy than high WMH $(p<0.0001)$ and low WMH $(p<$ 0.0001 ) older adults, and low WMH older adults having higher episodic accuracy than high WMH older adults $(p=0.012)$.

After confirming these group differences, we next sought to understand specific relationships between brain structure and cognition across the spectrum of cognitively normal aging, and all subsequent analyses were conducted on older adult data. Among older adult participants, the variables of age and global mean FA were continuous and normally distributed. Regressions of episodic memory performance on FA within older adults
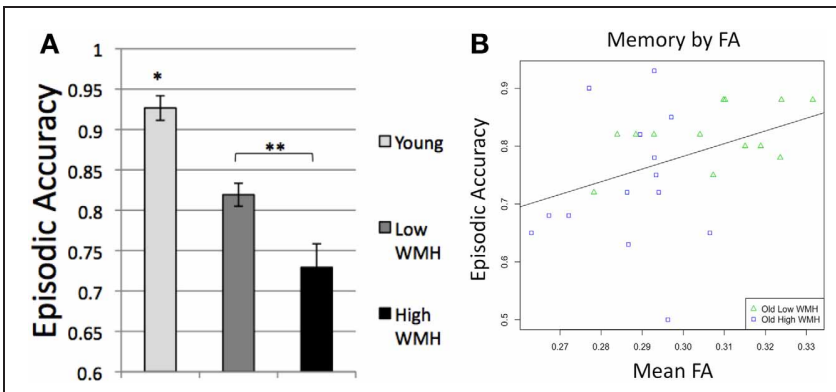

FIGURE 3 | (A) Episodic accuracy by group, *Differs from all groups, $p<0.0001,{ }^{* *}$ Groups differ, $p=0.012$; (B) Episodic accuracy by mean global white matter FA.
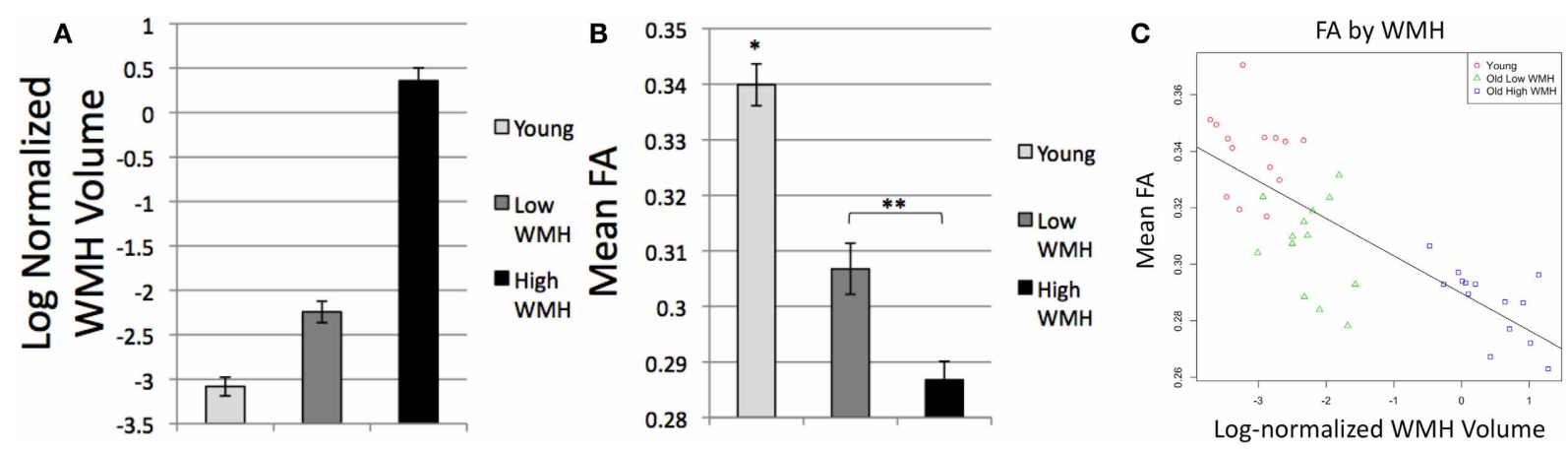

FIGURE 2 | (A) Global log-normalized WMH volume by group, all groups differ $p<0.0001$; (B) Mean global white matter FA by group, *Differs from all groups, $p<0.0001,{ }^{* *}$ Groups differ, $p=0.002$; (C) Mean global white matter FA by log-normalized WMH volume. 
demonstrated that reduced FA was associated with significantly decreased episodic accuracy $\left(R^{2}=0.15, p=0.046\right.$; Figure 3B $)$. This relationship, however, no longer remained significant $(p=$ 0.19 ) when controlling for age, sex, and education.

\section{VOXEL-WISE ASSOCIATIONS BETWEEN EPISODIC MEMORY, FLAIR, AND FA IN OLDER ADULTS}

We analyzed the relationships between episodic memory performance and voxel-wise FLAIR intensity and FA, among older adults, using the regression and cluster methods described above. This allowed us to assess the anatomical relationships between white matter injury or integrity and memory performance, independent of the contribution of age and other covariates, to inform our tract-specific analyses.

For our FLAIR intensity analysis, clusters of voxels in bilateral white matter were significantly and inversely related to episodic accuracy (Figure 4; left hemisphere cluster: $p=0.0056$; right hemisphere cluster: $p=0.0036$ ), independent of age, sex, and education. These clusters extended throughout the white matter of all four lobes, located predominantly within bilateral dorsal frontal, parietal, and subcortical white matter, as well as in bilateral temporal lobes, and right occipital lobe.

For our FA analysis, one significant FA cluster was noted in right posterior periventricular white matter (Figure 5; $p=0.047$ ) for a direct relation with memory performance. This cluster was located predominantly in right parietal white matter.

\section{EPISODIC MEMORY IS ASSOCIATED WITH WHITE MATTER INJURY TO SELECTED TRACTS IN OLDER ADULTS}

We further sought to identify which tracts among older adults demonstrated anatomically specific relationships between our white matter injury and integrity measures and memory performance, using the probabilistic tract atlas as described in the methods section. To accomplish this task, we first identified those tracts that passed through the clusters identified above (see Figure 6). For example, the inferior longitudinal fasciculus (ILF) overlapped with the clusters in the temporal lobes, while short association fibers connecting cuneus to superior occipital gyrus overlapped with the clusters in the right occipital lobe. For control purposes, we also examined tracts not contained within the identified area, such as corticospinal tract. These analyses were performed to identify where differences in white matter injury and integrity were predictive of impaired cognition, independent of the effects of age, sex, and education. We combined data from unilateral tract templates into bilateral measurements, and restricted our analyses to 10 tracts to minimize comparisons.

When examining bilateral tracts that intersected our clusters, we found that WMH in uncinate fasciculus and ILF were significantly inversely associated with memory performance, independent of age, sex, education, and tract FA (Table 2). FA was not associated with episodic memory performance in any of these models. Further analysis to examine the anatomic specificity of these findings found no relationship between episodic memory performance and WMH or FA values in corticospinal tract or in occipital short association fibers. When applying a less stringent threshold ( $p<0.05$ uncorrected), $\mathrm{WMH}$ in additional tracts, including the superior longitudinal fasciculus (frontalparietal portion), projections from the thalamus to the superior frontal gyrus and from the thalamus to the middle frontal gyrus, and the dorsal cingulum bundle, were inversely associated with memory performance independent of age, sex, education, and tract FA.

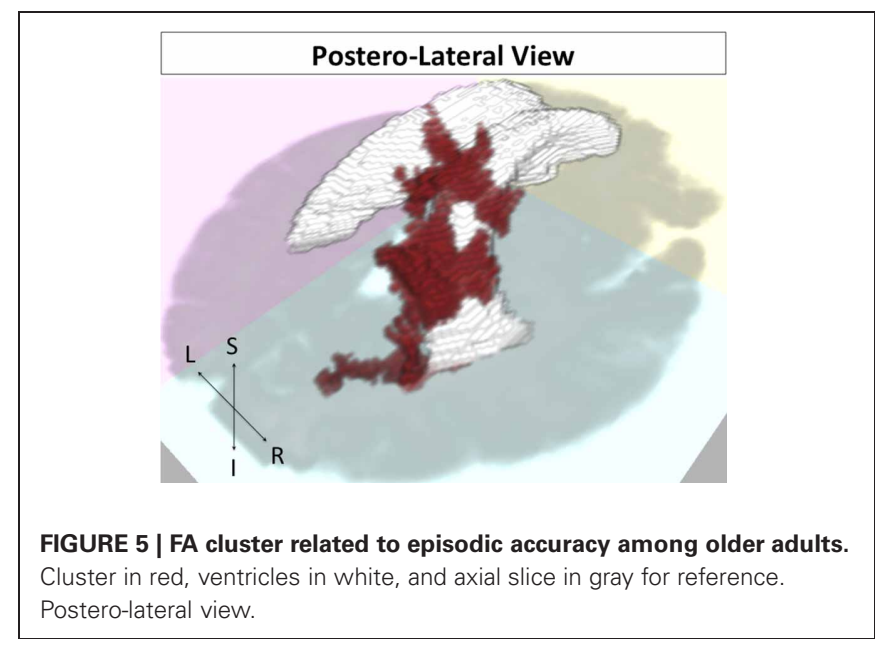

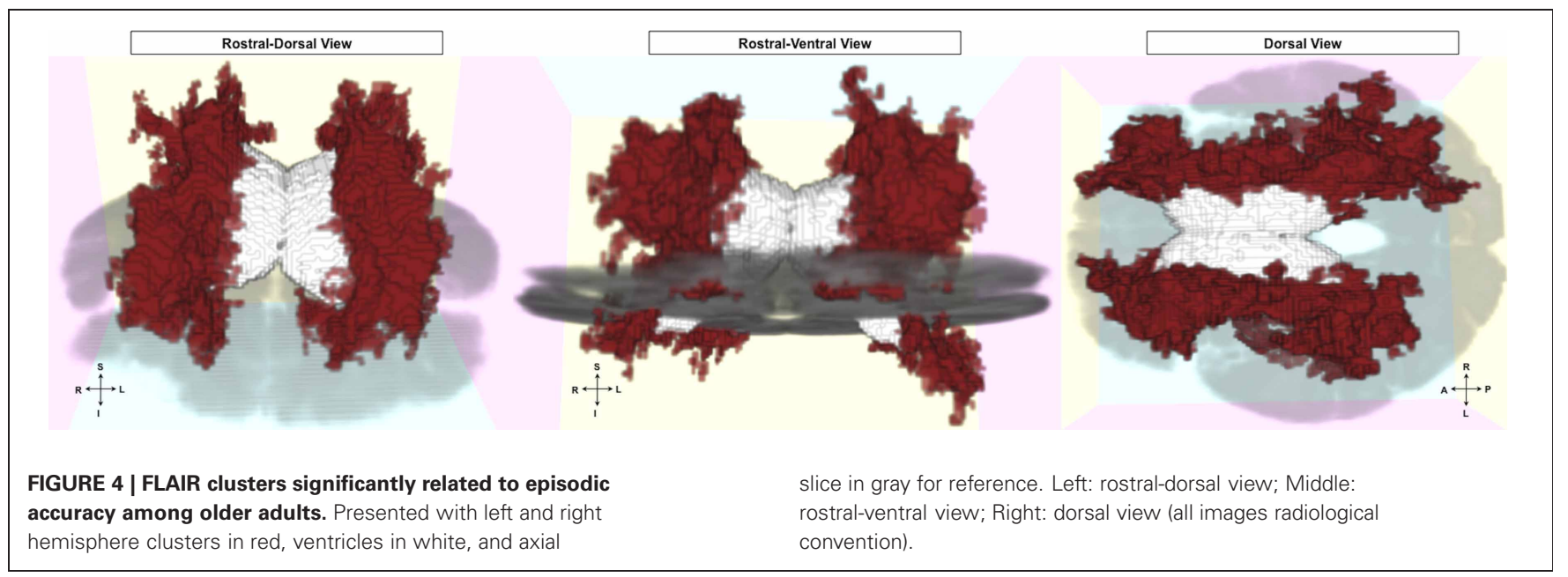



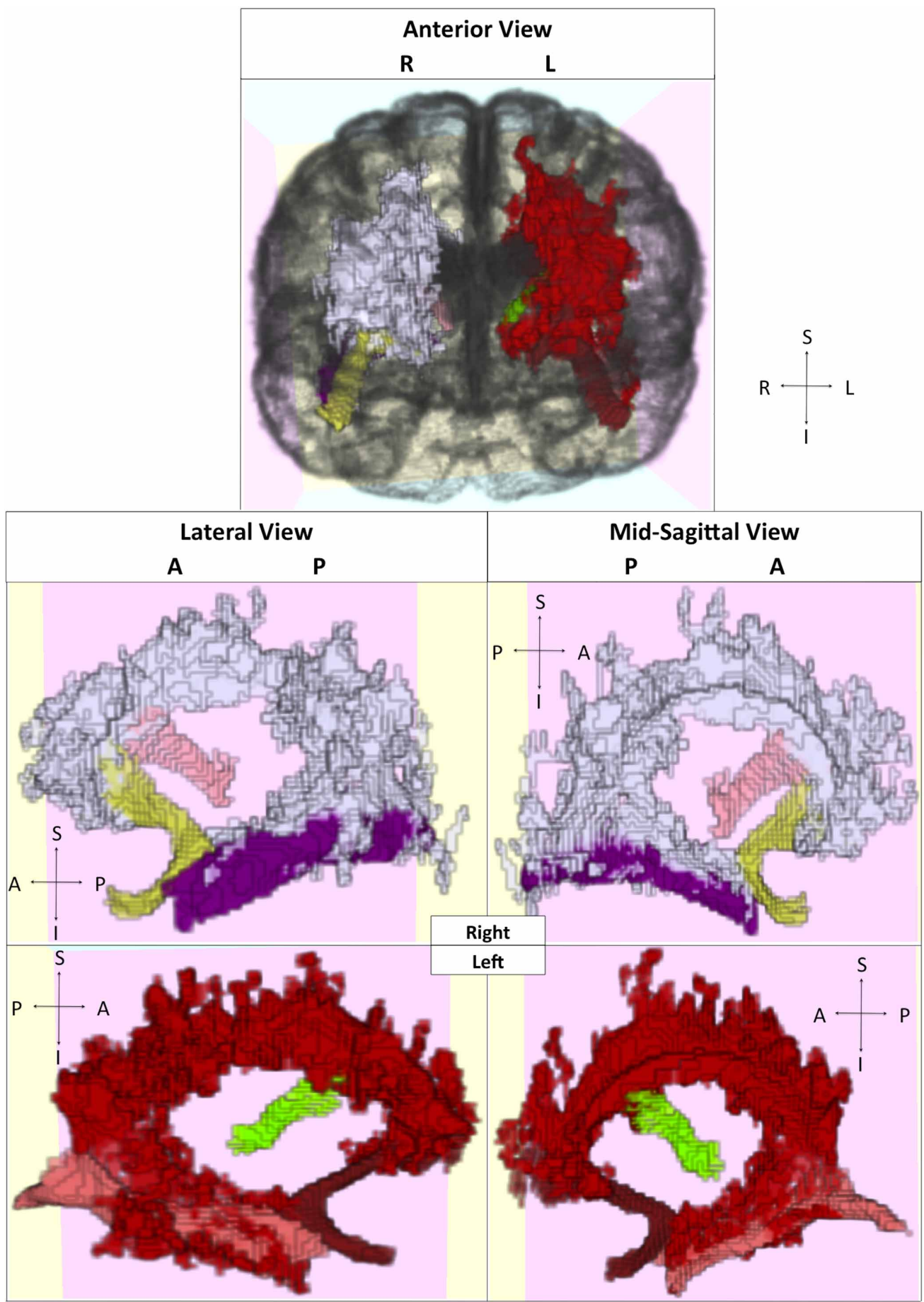

FIGURE 6 | Visualization of selected tracts passing through

clusters. Clusters and tracts displayed in different colors and presented in multiple orientations. White: right hemisphere cluster; Yellow: right uncinate fasciculus; Light pink: right thalamus-middle frontal gyrus projection; Purple: right inferior longitudinal fasciculus. Red: left hemisphere cluster; Dark red: left uncinate fasciculus; Green: left thalamus-middle frontal gyrus projection; Dark pink: left inferior longitudinal fasciculus.
We compared WMH effects to demonstrate the anatomic specificity of these tract-based findings. When comparing the contribution of ILF (a tract with a significant WMH effect) in regression models before and after adding covariates of corticospinal tract WMH and FA, we found ILF WMH remained a significant predictor of memory performance even after the inclusion of the additional covariates $(p=0.021$; independent of age, sex, and education, and ILF FA). We found a similar 
Table 2 | Tract-based multiple regression model $p$-values for WMH and FA covariates for 10 separate models.

\begin{tabular}{lll}
\hline & WMH & FA \\
\hline UNC & $0.0022^{* *}$ & 0.42 \\
SLF-FP & $0.013^{*}$ & 0.45 \\
TH-SFG & $0.010^{*}$ & 0.99 \\
TH-MFG & $0.0057^{*}$ & 0.64 \\
CST & 0.13 & 0.74 \\
CGH & 0.22 & 0.22 \\
CGC & $0.0052^{*}$ & 0.052 \\
ILF & $0.0038^{* *}$ & 0.55 \\
SAF-IOG-MOG & 0.15 & 0.69 \\
SAF-Cu-SOG & 0.09 & 0.88 \\
\hline
\end{tabular}

Model covariates include subject age, sex, and education, and tract WMH and FA as predictors of memory performance.

Abbreviations: UNC, uncinate fasciculus; SLF-FP, frontal-parietal portion of the superior longitudinal fasciculus; TH-SFG, projections between thalamus and superior frontal gyrus; TH-MFG, projections between thalamus and middle frontal gyrus; CST, corticospinal tract; CGH, ventral cingulum bundle; CGC, dorsal cingulum bundle; ILF, inferior longitudinal fasciculus; SAF-IOG-MOG, short association fibers between inferior occipital and middle occipital gyrus; SAF-CU-SOG, short association fibers between cuneus and superior occipital gyrus.

$* p<0.05$ (uncorrected);

${ }^{* *} p<0.0051$ ( $\alpha=0.05$ corrected for 10 comparisons).

Table 3 | Tract-based multiple regression model $p$-values for WMH and FA covariates as predictors of control cognitive measures.

\begin{tabular}{lllll}
\hline & ILF WMH & ILF FA & UNC WMH & UNC FA \\
\hline Forward digit span & 0.57 & 0.80 & 0.91 & 0.51 \\
Backward digit span & 0.62 & 0.43 & 0.72 & 0.18 \\
Category fluency & 0.66 & 0.42 & 0.22 & 0.60
\end{tabular}

Model covariates include subject age, sex, and education, and tract WMH and FA. All values not significant at $p<0.05$.

Abbreviations: UNC, uncinate fasciculus; ILF, inferior longitudinal fasciculus.

preservation, in a separate model, of the effect of uncinate WMH after adding corticospinal tract white matter measures (new uncinate WMH $p=0.006$ ). Further comparison of WMH effects demonstrated differences in the strength of association among these different tracts. When both uncinate WMH and ILF WMH were entered into a model to predict cognition (independent of age, sex, and education), uncinate WMH remained significant $(p=0.03)$ while ILF WMH was only marginally significant $(p=0.06)$.

To further demonstrate the specificity of these findings, we tested whether performance on other behavioral measures was associated with tract white matter injury and integrity. We found that neither WMH nor FA for the significant tracts (uncinate fasciculus or ILF) were significant predictors of performance on forward digit span, backward digit span, or category fluency (Table 3). These results suggest that episodic memory performance depends on the integrity of multiple specific white matter pathways.

\section{DISCUSSION}

The goal of the present study was to understand whether white matter changes in specific pathways are critical for impairing episodic memory performance in normal cognitive aging, and whether changes in these tracts related to white matter injury or to microstructural alterations in white matter integrity are most strongly associated with performance. The present results suggest that multiple pathways are important, but that specific connections of frontal, temporal, and parietal cortex and frontalsubcortical white matter tracts were most affected. Regressions of performance on white matter measures consistently demonstrated inverse associations between white matter injury and episodic memory at multiple levels of structural analysis. Results from our regression cluster analyses suggest that localized injury to white matter connections between the frontal, temporal, and parietal cortex may contribute to episodic source memory impairments in cognitively normal older adults independent of age, suggesting that at least some of age-related memory performance differences might reflect clinically silent white matter injury (Buckner, 2004). Further, tract analyses demonstrated significant associations between memory performance and white matter injury to selected fiber tracts, including uncinate fasciculus, ILF, projections from the thalamus to the superior frontal gyrus and to the middle frontal gyrus, superior longitudinal fasciculus, and dorsal cingulum bundle. WMH in these tracts were most strongly associated with episodic memory function, suggesting that apparent injury is necessary to cause cognitive differences rather than the more subtle changes in white matter fiber integrity identified by FA. We also demonstrated that white matter integrity in these tracts was not associated with performance on fluency and working memory tasks, supporting the idea that localized injury to tracts can have selective effects on cognition. This pattern of results strongly suggest that episodic source memory performance in older adults is particularly vulnerable to injury to connections between the frontal, temporal, and parietal cortex and their targets, as well as frontal-subcortical connections.

While the existing literature has shown that frontal white matter integrity is critical for episodic memory, previous studies, including some from our laboratory, relating white matter structural changes to age-related differences in episodic memory performance have not identified which specific white matter tracts are most involved in network disconnection. The links demonstrated here between memory and multiple, distinct measures of white matter integrity in specific fiber tracts, many of them frontally anchored, represents an empirical advance in the understanding of how cognitive dysfunction may arise from age-related network disconnection. This study is, to our knowledge, the first to test both DTI and WMH age-related differences related to cognition in the same sample using data from specific white matter tracts; however, these are associations in a small cross-sectional study and further testing is required.

We consistently found that FA was not as strongly associated with memory performance as WMH. White matter tract FA did not contribute significantly to any regression models above tract $\mathrm{WMH}$, suggesting that the tract WMH measures were a much more robust predictor of cognitive performance. Recent research suggests that FA reductions surround $\mathrm{WMH}$, but that WMH best 
represent "core" injury (Maillard et al., 2011). It is possible, therefore, that FA reductions represent more subtle damage to white matter tracts, and do not signify the same degree of functional disconnection as WMH. These results suggest that white matter injury to selected tracts, above and beyond microstructural changes in white matter integrity, is necessary to cause differences in memory performance among older adults. This injury is agnostic to the orientation of specific white matter tracts, and at most a portion of certain tracts pass through areas of injury. However, as these tracts pass through these regions and can be affected by injury, we can use the patterns of white matter injury among older adults to demonstrate how injury to specific tracts is associated with behavior.

The cognitive changes observed here may represent the anatomically localized injury of white matter tracts that connect circuits necessary for successful memory encoding and retrieval processes. Traditionally, episodic memory function has been attributed to the structure and function of the hippocampus and the medial temporal lobes (Zola-Morgan and Squire, 1993), and age-related differences in memory performance to changes in temporal structures (Tisserand and Jolles, 2003). Our finding that frontal-temporal connectivity (e.g., uncinate fasciculus) and connectivity of temporal with parietal and occipital cortex (e.g., ILF) is associated with episodic source memory performance in aging supports these concepts. However, recent studies of age-related memory differences have demonstrated that structural and functional changes in other brain systems, including frontal-subcortical and frontal-parietal systems, are associated with differences in memory performance in cognitively normal older individuals (Buckner, 2004; Dickerson and Eichenbaum, 2010; Bollinger et al., 2011). Changes in the integrity of white matter connections of the frontal cortex with its targets appear to be critical to age-related memory differences. For example, not only is prefrontal white matter integrity associated with memory performance (Bucur et al., 2008; Head et al., 2008), but increasing WMH volume also is associated with decreased PFC recruitment during episodic memory tasks (Nordahl et al., 2006). The current findings are in agreement with previous reports (Buckner, 2004; Smith et al., 2011) that suggest disruption of connectivity in prefrontal-subcortical (e.g., thalamus to frontal cortex connections) and prefrontal-parietal (e.g., superior longitudinal fasciculus) circuits is associated with age-related memory dysfunction.

We also found that injury to the dorsal cingulum bundle was associated with episodic memory impairment. Fibers of the dorsal cingulum bundle travel widely, but are primarily known to connect frontal cortex with the inferior parietal lobule, while more ventral aspects (which were not found to be significant in this study) connect with medial temporal lobe structures (Schmahmann et al., 2007). The cingulum bundle has been associated with mnemonic processing, among other functions, due to these connections between frontal and temporal areas important for episodic memory performance (Schmahmann and Pandya, 2006). In addition, the significant FA-memory cluster we found in older adults in right parietal white matter partially overlapped with the posterior aspect of the right dorsal cingulum bundle, in keeping with previous findings that greater memory impairment in older adults was associated with lower FA in the posterior cingulum bundle (Kantarci et al., 2011).

The uncinate fasciculus connects rostral inferior temporal regions known to be important for object recognition, medial temporal areas important to recognition memory, and medial, orbital and prefrontal cortices important in inhibition and self-regulation, and in primate studies has been shown to be critical to successful visual learning (Schmahmann et al., 2007). Our finding that uncinate injury is associated with source memory performance on this task is in agreement with these previous anatomical and behavioral studies.

The ILF courses extensively through the white matter of the temporal lobes, projecting to regions such as temporal pole and superior temporal sulcus, with caudal branches extending vertically into inferior parietal lobe and horizontally into inferior temporal and occipital regions (Schmahmann et al., 2007). Recent research suggests that ILF integrity may be important to successful visual memory function (Davis et al., 2009; Shinoura et al., 2007); such evidence supports our finding that ILF injury is strongly associated with source memory performance on a task with a strong visual component. Given that occipital fibers did not show significant associations with cognition, the temporoparietal connections of the ILF may be more critical to memory performance than temporo-occipital connections; however, this requires further testing.

Evidence exists in the neuroanatomical literature for multiple parallel frontal-striatal loops, which mediate behaviors ranging from action selection to executive control and memory (Alexander et al., 1986). One such circuit-beginning in dorsolateral prefrontal cortex (DLPFC) - projects serially to caudate nucleus, globus pallidus, thalamus, and back to DLPFC. Disruption of this circuit at any point in the pathway, including not only frontal-striatal connections, but also outflow tracts connecting thalamic nuclei to frontal cortex, may disrupt attention, planning, and organization (Cummings, 1993) and thereby impact memory encoding and retrieval strategies. The thalamocortical outflow tracts identified in our analyses are in the same general area as fiber tracts known to connect ventral anterior and medial dorsal thalamic nuclei with middle and superior frontal gyri (areas 9/46d and 9/46v) in macaque and human studies (Petrides and Pandya, 2002; Schmahmann and Pandya, 2006). These tracts also appear to pass through the cluster that demonstrated a significant association between white matter injury and memory performance. Furthermore, the thalamus is situated at the intersection of frontal-subcortical and medial temporal-limbic circuits involving the hippocampus (Cummings, 1993); reduced thalamo-frontal connectivity, as observed here, may impair communication between brain systems in the performance of memory tasks.

In the older adult sample tested here, impairment of frontalsubcortical circuits, particularly the DLPFC-subcortical circuit, due to white matter injury may specifically influence frontal systems through impaired connectivity with subcortical systems, with subsequent executive dysfunction leading to impaired episodic memory performance by impairing memory encoding or retrieval. WMH are thought to play an important role in memory tests that rely on executive control processes (Parks et al., 
2010). Previous research suggests that dysfunctional maintenance and manipulation of information in working memory may result in deficits in episodic encoding and retrieval processes (Nordahl et al., 2005). Therefore, impairment of executive control processes important to working memory may contribute to age-related episodic memory differences (Parks et al., 2011).

Not only does white matter injury as measured by WMH affect frontal-subcortical systems, but the degree of impairment among older subjects with extensive WMH is sufficient to be clinically relevant as well. For example, high WMH subjects performed more than one standard deviation below low WMH subjects on the memory task, and poorer in most neuropsychological measures. This result is consistent with previous data suggesting that WMH may be sufficient to cause clinically relevant memory loss and the diagnosis of mild cognitive impairment (Nordahl et al., 2005).

Neuroanatomical and electrophysiological studies have revealed reciprocal white matter connections between prefrontal and parietal cortex that are part of networks important to attentional control, visual spatial processing, and memory performance (Goldman-Rakic, 1987; Selemon and Goldman-Rakic, 1988). Recent models of attention argue that dorsal frontal and parietal regions interact in the top-down control of attention not only for perception but also for memory retrieval guidance, whereby attentional selection and implementation are guided by retrieval goals (Corbetta and Shulman, 2002; Cabeza et al., 2008). Indeed, recent theories of episodic retrieval have argued that dorsal parietal cortex, in conjunction with dorsal frontal regions, performs top-down attentional control of episodic retrieval, biasing medial temporal lobe activity, in a manner similar to the attentional control of sensory perceptual regions (Cabeza, 2008). fMRI studies support this idea of parietal involvement in memory retrieval (Cabeza et al., 2011). Control processes mediated by frontal-parietal networks, therefore, may be critical to successful episodic memory performance. The current study, consistent with these theories, demonstrates that injury to prefrontal-parietal white matter tracts such as the superior longitudinal fasciculus may be as effective in producing cognitive impairments as lesions in the gray matter (Raz et al., 1998) among older individuals with otherwise normal cognitive function. However, the episodic memory paradigm in the current study was unable to disambiguate whether injury is associated specifically with encoding or retrieval processes, or both.

The present results are consistent with previous studies suggesting that episodic memory performance depends on the integrity of white matter tracts between frontal cortex and subcortical structures, and between frontal and temporo-parietal cortex (Nordahl et al., 2006; Schott et al., 2011; Smith et al., 2011). Additionally, our results are in agreement with studies linking PFC damage and network disconnection with impaired source memory accuracy (Janowsky et al., 1989; Duarte et al., 2005; Swick et al., 2006). Significant inverse relationships of WMH volume with episodic memory and executive control have been previously demonstrated in other cognitive aging samples (Gunning-Dixon and Raz, 2000; Nordahl et al., 2006). In addition, among the same subjects used in this experiment, significant associations were found between $\mathrm{WMH}$ and executive control impairments (Mayda et al., 2011), confirming a similar relationship between WMH, memory and executive control among individuals diagnosed with mild cognitive impairment (Nordahl et al., 2005). Together, these results support the idea that increased WMH volume may increase risk for long-term executive control impairments, which may be leading to the memory dysfunction we observed (Parks et al., 2011).

The current study has several potential limitations. Null results regarding certain relationships may be due to multiple factors including sample heterogeneity in white matter changes, relatively low subject numbers, and imaging resolution limitations. The failure to identify significant FA-memory relationships may demonstrate the need for higher-resolution DTI protocols; perhaps a greater number of gradient directions were necessary to provide more sensitive measures of microstructural white matter differences. In addition our results may suggest the need for more anatomically constrained hypotheses of FA-cognition relations; future advances may allow for testing of relationships between reduced local FA (e.g., of the fornix) and impaired episodic memory. It is unlikely that the greater relation of WMH than FA to memory performance once age, sex, and education are controlled is due to correlations between FA and individual ability factors such as education, because FA was not significantly correlated with education in our sample $(p=0.10)$ and removal of education from the tract analyses did not alter the results. Further, while we collapsed tract-specific white matter measures bilaterally to conserve statistical power, lateralized effects may have been present in the data. Additionally, others have demonstrated relatively robust correlations in older adults between FA and cognitive measures, such as working memory (e.g., Zahr et al., 2009); it is possible, although unlikely, that the weaker association between memory and FA we have demonstrated here may be specific to our source memory paradigm, rather than due to greater sensitivity of performance to $\mathrm{WMH}$.

In conclusion, white matter injury as measured by $\mathrm{WMH}$ may specifically influence brain systems, particularly frontal and temporal systems, through impaired network connectivity in the performance of an episodic memory task. The presence of white matter injury is related to episodic memory function at multiple levels of structural analysis. Patterns of association between white matter and episodic memory point to a pattern whereby episodic memory performance depends on white matter integrity of multiple regions. Such findings corroborate and extend previous findings from our laboratory by making links between memory and specific white matter tracts. These data support the hypothesis that age- and CVD-related white matter injury lead to disconnection of cortical areas. This disconnection may provide a neural mechanism to explain the observed age-related cognitive impairments among older individuals with WMH. We seek to understand the mechanisms by which increased age and CVD, which increases the extent of WMH, may affect brain structure and cognition across a broad spectrum of older adults.

\section{ACKNOWLEDGMENTS}

This work was supported by National Institute on Aging grants P30 AG10129, R01 AG 10220, and R01 AG021028 (to Charles DeCarli). 


\section{REFERENCES}

Alexander, G. E., Delong, M. R., and Strick, P. L. (1986). Parallel organization of functionally segregated circuits linking basal ganglia and cortex. Annu. Rev. Neurosci. 9, 357-381.

Andrews-Hanna, J. R., Snyder, A. Z., Vincent, J. L., Lustig, C., Head, D., Raichle, M. E., and Buckner, R. L. (2007). Disruption of large-scale brain systems in advanced aging. Neuron 56, 924-935.

Assaf, Y., and Pasternak, O. (2008). Diffusion tensor imaging (DTI)based white matter mapping in brain research: a review. J. Mol. Neurosci. 34, 51-61.

Basser, P. J., and Pierpaoli, C. (1998). A simplified method to measure the diffusion tensor from seven MR images. Magn. Reson. Med. 39, 928-934.

Bollinger, J., Rubens, M. T., Masangkay, E., Kalkstein, J., and Gazzaley, A. (2011). An expectationbased memory deficit in aging. Neuropsychologia 49, 1466-1475.

Buckner, R. L. (2004). Memory and executive function in aging and $\mathrm{AD}$ : multiple factors that cause decline and reserve factors that compensate. Neuron 44, 195-208.

Bucur, B., Madden, D. J., Spaniol, J., Provenzale, J. M., Cabeza, R., White, L. E., and Huettel, S. A. (2008). Agerelated slowing of memory retrieval: contributions of perceptual speed and cerebral white matter integrity. Neurobiol. Aging 29, 1070-1079.

Cabeza, R. (2002). Hemispheric asymmetry reduction in older adults: the HAROLD model. Psychol. Aging 17, 85-100.

Cabeza, R. (2008). Role of parietal regions in episodic memory retrieval: the dual attentional processes hypothesis. Neuropsychologia 46, 1813-1827.

Cabeza, R., Ciaramelli, E., Olson, I. R., and Moscovitch, M. (2008). The parietal cortex and episodic memory: an attentional account. Nat. Rev. Neurosci. 9, 613-625.

Cabeza, R., Mazuz, Y. S., Stokes, J., Kragel, J. E., Woldorff, M. G., Ciaramelli, E., Olson, I. R., and Moscovitch, M. (2011). Overlapping parietal activity in memory and perception: evidence for the attention to memory model. J. Cogn. Neurosci. 23, 3209-3217.

Cabeza, R., and Nyberg, L. (2000). Neural bases of learning and memory: functional neuroimaging evidence. Curr. Opin. Neurol. 13, 415-421.

Cavada, C., and Goldman-Rakic, P. S. (1989). Posterior parietal cortex in rhesus monkey: II. Evidence for segregated corticocortical networks linking sensory and limbic areas with the frontal lobe. J. Comp. Neurol. 287, 422-445.

Corbetta, M., and Shulman, G. L. (2002). Control of goal-directed and stimulus-driven attention in the brain. Nat. Rev. Neurosci. 3, 201-215.

Cummings, J. L. (1993). Frontalsubcortical circuits and human behavior. Arch. Neurol. 50, 873-880.

Davis, S. W., Dennis, N. A., Buchler, N. G., White, L. E., Madden, D. J. and Cabeza, R. (2009). Assessing the effects of age on long white matter tracts using diffusion tensor tractography. Neuroimage 46, 530-541.

DeCarli, C., Fletcher, E., Ramey, V., Harvey, D., and Jagust, W. J. (2005a). Anatomical mapping of white matter hyperintensities (WMH): exploring the relationships between periventricular $\mathrm{WMH}$, deep WMH, and total WMH burden. Stroke 36, 50-55.

DeCarli, C., Maisog, J., Murphy, D. G., Teichberg, D., Rapoport, S. I., and Horwitz, B. (1992). Method for quantification of brain, ventricular, and subarachnoid CSF volumes from MR images. J. Comput. Assist. Tomogr. 16, 274-284.

DeCarli, C., Massaro, J., Harvey, D., Hald, J., Tullberg, M., Au, R., Beiser, A., D'Agostino, R., and Wolf, P. A. (2005b). Measures of brain morphology and infarction in the Framingham heart study: establishing what is normal. Neurobiol. Aging 26, 491-510.

DeCarli, C., Murphy, D. G., Teichberg, D., Campbell, G., and Sobering, G. S. (1996). Local histogram correction of MRI spatially dependent image pixel intensity nonuniformity. J. Magn. Reson. Imaging 6, 519-528.

DeCarli, C., Murphy, D. G., Tranh, M., Grady, C. L., Haxby, J. V., Gillette, J. A., Salerno, J. A., Gonzales-Aviles, A., Horwitz, B., and Rapoport, S. I. (1995). The effect of white matter hyperintensity volume on brain structure, cognitive performance, and cerebral metabolism of glucose in 51 healthy adults. Neurology 45, 2077-2084.

DeCarli, C., Reed, B. R., Jagust, W., Martinez, O., Ortega, M., and Mungas, D. (2008). Brain behavior relationships among African Americans, whites, and Hispanics. Alzheimer Dis. Assoc. Disord. 22, 382-391.

Dickerson, B. C., and Eichenbaum, H. (2010). The episodic memory system: neurocircuitry and disorders.
Neuropsychopharmacology 35, 86-104.

Duarte, A., Ranganath, C., and Knight, R. T. (2005). Effects of unilateral prefrontal lesions on familiarity, recollection, and source memory. J. Neurosci. 25, 8333-8337.

Dulas, M. R., and Duarte, A (2012). The effects of aging on material-independent and materialdependent neural correlates of source memory retrieval. Cereb. Cortex 22, 37-50.

Glisky, E. L., Rubin, S. R., and Davidson, P. S. R. (2001). Source memory in older adults: an encoding or retrieval problem? J. Exp. Psychol. Learn. Mem. Cogn. 27, 1131.

Goldman-Rakic, P. S. (1987). Circuitry of the frontal association cortex and its relevance to dementia. Arch. Gerontol. Geriatr. 6, 299-309.

Grady, C. L., and Craik, F. I. (2000). Changes in memory processing with age. Curr. Opin. Neurobiol. 10 224-231.

Gunning-Dixon, F. M., and Raz, N. (2000). The cognitive correlates of white matter abnormalities in normal aging: a quantitative review. Neuropsychology 14, 224-232.

Head, D., Rodrigue, K. M., Kennedy, K. M., and Raz, N. (2008) Neuroanatomical and cognitive mediators of age-related differences in episodic memory. Neuropsychology 22, 491-507.

Jacobs, H. I. L., Leritz, E. C., Williams V. J., Van Boxtel, M. P. J., Elst, W. V. D., Jolles, J., Verhey, F. R. J. McGlinchey, R. E., Milberg, W. P., and Salat, D. H. (2011). Association between white matter microstructure, executive functions, and processing speed in older adults: the impact of vascular health. Hum. Brain Mapp. doi: 10.1002/ hbm.21412. [Epub ahead of print].

Janowsky, J. S., Shimamura, A. P., and Squire, L. R. (1989). Source memory impairment in patients with frontal lobe lesions. Neuropsychologia 27, 1043-1056.

Kantarci, K., Senjem, M., Avula, R., Zhang, B., Samikoglu, A. R., Weigand, S. D., Przybelski, S. A., Edmonson, H., Vemuri, P., Knopman, D. S., Boeve, B. F., Ivnik, R. J., Smith, G. E., Petersen, R. C., and Jack, C. R. (2011). Diffusion tensor imaging and cognitive function in older adults with no dementia. Neurology 77, 26-34.

Lee, D. Y., Fletcher, E., Martinez, O., Zozulya, N., Kim, J., Tran, J., Buonocore, M., Carmichael, O., and DeCarli, C. (2010). Vascular and degenerative processes differentially affect regional interhemispheric connections in normal aging, mild cognitive impairment, and Alzheimer disease. Stroke 41, 1791-1797.

Maillard, P., Fletcher, E., Harvey, D. Carmichael, O., Reed, B., Mungas, D., and DeCarli, C. (2011). White matter hyperintensity penumbra. Stroke 42, 1917-1922.

Mayda, A. B. V., Westphal, A., Carter, C. S., and DeCarli, C. (2011). Late life cognitive control deficits are accentuated by white matter disease burden. Brain 134, 1673-1683.

Mori, S., Wakana, S., and Van Zijl, P. C. M. (2005). MRI Atlas of Human White Matter. Amsterdam: Elsevier.

Nichols, T. E., and Holmes, A. P. (2002). Nonparametric permutation tests for functional neuroimaging: a primer with examples. Hum. Brain Mapp. 15, 1-25.

Nordahl, C. W., Ranganath, C., Yonelinas, A. P., DeCarli, C. Fletcher, E., and Jagust, W. J. (2006). White matter changes compromise prefrontal cortex function in healthy elderly individuals. J. Cogn Neurosci. 18, 418-429.

Nordahl, C. W., Ranganath, C., Yonelinas, A. P., DeCarli, C., Reed, B. R., and Jagust, W. J. (2005). Different mechanisms of episodic memory failure in mild cognitive impairment. Neuropsychologia 43, 1688-1697.

Parks, C. M., DeCarli, C., Jacoby, L. L., and Yonelinas, A. P. (2010). Aging effects on recollection and familiarity: the role of white matter hyperintensities. Neuropsychol. Dev. Cogn. B Aging Neuropsychol. Cogn. 17, 422-438.

Parks, C. M., Iosif, A.-M., Farias, S., Reed, B., Mungas, D., and DeCarli, C. (2011). Executive function mediates effects of white matter hyperintensities on episodic memory. Neuropsychologia 49, 2817-2824.

Petrides, M., and Pandya, D. N. (2002). Comparative cytoarchitectonic analysis of the human and the macaque ventrolateral prefrontal cortex and corticocortical connection patterns in the monkey. Eur. J. Neurosci. 16, 291-310.

Pfefferbaum, A., Sullivan, E. V., Hedehus, M., Lim, K. O., Adalsteinsson, E., and Moseley, M. (2000). Age-related decline in brain white matter anisotropy measured with spatially corrected echo-planar diffusion tensor imaging. Magn. Reson. Med. 44, 259-268.

R Development Core Team (2011). $R$ : A language and environment for statistical computing. Vienna, 
Austria: R Foundation for Statistical Computing.

Rajah, M. N., Languay, R., and Valiquette, L. (2010). Age-related changes in prefrontal cortex activity are associated with behavioural deficits in both temporal and spatial context memory retrieval in older adults. Cortex 46, 535-549.

Raz, N., Gunning-Dixon, F. M., Head, D., Dupuis, J. H., and Acker, J. D. (1998). Neuroanatomical correlates of cognitive aging: evidence from structural magnetic resonance imaging. Neuropsychology 12, 95-114.

Salthouse, T. A. (2009). When does age-related cognitive decline begin? Neurobiol. Aging 30, 507-514.

Schmahmann, J. D., and Pandya, D. N. (2006). Fiber Pathways of the Brain. New York, NY: Oxford University Press.

Schmahmann, J. D., Pandya, D. N., Wang, R., Dai, G., D’Arceuil, H. E., de Crespigny, A. J., and Wedeen, V. J. (2007). Association fibre pathways of the brain: parallel observations from diffusion spectrum imaging and autoradiography. Brain $130,630-653$.

Schott, B. H., Niklas, C., Kaufmann, J., Bodammer, N. C., Machts, J., Schütze, H., and Düzel, E. (2011). Fiber density between rhinal cortex and activated ventrolateral prefrontal regions predicts episodic memory performance in humans. Proc. Natl. Acad. Sci. U.S.A. 108, 5408-5413.
Selemon, L. D., and Goldman-Rakic, P. S. (1988). Common cortical and subcortical targets of the dorsolateral prefrontal and posterior parietal cortices in the rhesus monkey: evidence for a distributed neural network subserving spatially guided behavior. J. Neurosci. 8, 4049-4068.

Shinoura, N., Suzuki, Y., Tsukada, M., Katsuki, S., Yamada, R., Tabei, Y., Saito, K., and Yagi, K. (2007). Impairment of inferior longitudinal fasciculus plays a role in visual memory disturbance. Neurocase 13 , 127-130.

Smith, E. E., Salat, D. H., Jeng, J., McCreary, C. R., Fischl, B., Schmahmann, J. D., Dickerson, B. C., Viswanathan, A., Albert, M. S., Blacker, D., and Greenberg, S. M. (2011). Correlations between MRI white matter lesion location and executive function and episodic memory. Neurology 76, 1492-1499.

Swick, D., Senkfor, A. J., and Van Petten, C. (2006). Source memory retrieval is affected by aging and prefrontal lesions: behavioral and ERP evidence. Brain Res. 1107, 161-176.

Tisserand, D. J., and Jolles, J. (2003). On the involvement of prefrontal networks in cognitive ageing. Cortex 39 , 1107-1128.

Vernooij, M. W., De Groot, M., Van Der Lugt, A., Ikram, M. A., Krestin, G. P., Hofman, A., Niessen, W. J., and Breteler, M. M. B. (2008). White matter atrophy and lesion formation explain the loss of structural integrity of white matter in aging. Neuroimage 43, 470-477.

West, R. L. (1996). An application of prefrontal cortex function theory to cognitive aging. Psychol. Bull. 120, 272-292.

Yeterian, E. H., Pandya, D. N. Tomaiuolo, F., and Petrides, M. (2012). The cortical connectivity of the prefrontal cortex in the monkey brain. Cortex 48, 58-81.

Yonelinas, A. P., Hopfinger, J. B., Buonocore, M. H., Kroll, N. E., and Baynes, K. (2001) Hippocampal, parahippocampal and occipital-temporal contributions to associative and item recognition memory: an fMRI study. Neuroreport 12, 359-363.

Zahr, N. M., Rohlfing, T., Pfefferbaum, A., and Sullivan, E. V. (2009). Problem solving, working memory, and motor correlates of association and commissural fiber bundles in normal aging: a quantitative fiber tracking study. Neuroimage 44 , 1050-1062.

Zhang, Y., Zhang, J., Oishi, K., Faria, A. V., Jiang, H., Li, X., Akhter, K., RosaNeto, P., Pike, G. B., Evans, A., Toga, A. W., Woods, R., Mazziotta, J. C., Miller, M. I., Van Zijl, P. C. M., and Mori, S. (2010). Atlas-guided tract reconstruction for automated and comprehensive examination of the white matter anatomy. Neuroimage 52, 1289-1301.

Ziegler, D. A., Piguet, O., Salat, D H., Prince, K., Connally, E., and Corkin, S. (2010). Cognition in healthy aging is related to regional white matter integrity, but not cortical thickness. Neurobiol. Aging 31, 1912-1926.

Zola-Morgan, S., and Squire, L. R. (1993). Neuroanatomy of memory. Annu. Rev. Neurosci. 16, 547-563.

Conflict of Interest Statement: The authors declare that the research was conducted in the absence of any commercial or financial relationships that could be construed as a potential conflict of interest.

Received: 27 October 2011; paper pending published: 23 November 2011; accepted: 01 March 2012; published online: 16 March 2012

Citation: Lockhart SN, Mayda ABV Roach AE, Fletcher E, Carmichael $O$, Maillard P, Schwarz CG, Yonelinas AP, Ranganath C and DeCarli C (2012) Episodic memory function is associated with multiple measures of white matter integrity in cognitive aging. Front. Hum. Neurosci. 6:56. doi: 10.3389/ fnhum. 2012.00056

Copyright () 2012 Lockhart, Mayda, Roach, Fletcher, Carmichael, Maillard, Schwarz, Yonelinas, Ranganath and DeCarli. This is an open-access article distributed under the terms of the Creative Commons Attribution Non Commercial License, which permits non-commercial use, distribution, and reproduction in other forums, provided the original authors and source are credited. 